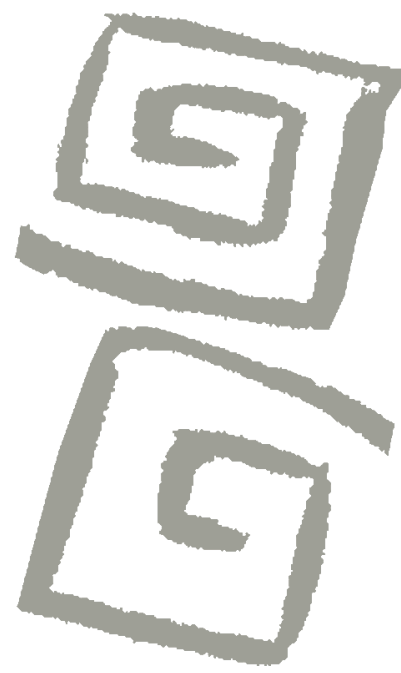

\title{
Colaboración interprofesional para el seguimiento odontológico prenatal en atención primaria de la salud
}

\author{
Interprofessional collaboration for prenatal dental follow \\ up in primary health care
}

Mirelle Varela Rodrigues Bandeira ${ }^{1}$, Adriano da Costa Belarmino², Saiwori de Jesus Silva Bezerra dos Anjos ${ }^{3}$, Maria Rocineide Ferreira da Silva ${ }^{4}$, Antonio Rodrigues Ferreira Junior ${ }^{5}$

${ }^{1}$ Cirujana dentista. Magíster en Salud Colectiva. Secretaría Municipal de Salud, Fortaleza, Ceará, Brasil $\triangle$ (iD)

${ }^{2}$ Enfermero. Especialista en Enfermería Obstétrica y Neonatal. Universidade Estadual do Ceará, Fortaleza, Ceará, Brasil. $\triangle$ (iD)

${ }^{3}$ Enfermera. Doctora en Enfermería. Docente, Universidade Estadual do Ceará, Fortaleza, Ceará, Brasil. $\triangle$ iD

${ }^{4}$ Enfermera. Doctora en Salud Colectiva. Docente Universidade Estadual do Ceará, Brasil. $\bowtie$ (iD

${ }^{5}$ Enfermero. Doctor en Salud Colectiva. Docente, Universidade Estadual do Ceará, Brasil. $\bowtie$ iD
RESUMEN Con el objetivo de comprender las prácticas colaborativas al interior de los equipos interprofesionales para el seguimiento odontológico prenatal en atención primaria de la salud, se realizó un estudio cualitativo exploratorio descriptivo con trabajadores directamente implicados en el cuidado prenatal del municipio de Fortaleza, Ceará, Brasil. Para ello, entre junio y agosto de 2018, se Ilevaron a cabo 24 entrevistas individuales semiestructuradas en profundidad; la información obtenida se organizó aplicando el análisis temático, la fundamentación teórica y la interpretación se sustentan en la hermenéutica dialéctica. Los discursos revelaron tres categorías: percepciones profesionales acerca de la práctica colaborativa, desafíos de la colaboración interprofesional y prácticas de colaboración en el equipo. Se verificó una percepción favorable de los profesionales acerca de la colaboración interprofesional en el cuidado materno-infantil; sin embargo, en algunos casos, la práctica laboral interdisciplinaria dista de efectivizarse por la existencia de barreras que dificultan su concreción.

PALABRAS CLAVES Atención Prenatal; Relaciones Interprofesionales; Atención Primaria de la Salud; Brasil.

\begin{abstract}
The aim of this study is to understand interprofessional teams' collaborative practices for prenatal dental follow-up in primary health care. An exploratory, qualitative and descriptive study was carried out, in which workers directly involved in prenatal care in the city of Fortaleza, Brazil were included. A total of 24 individual semi structured in depth interviews were conducted between June and August of 2018. The information was organized based on a thematic analysis, and the hermeneutic dialectic method was used for the theoretical foundation and interpretation. Three discursive categories were identified: professional perceptions about collaborative practice, challenges in interprofessional collaboration, and collaboration practices within the team. The professionals' perception about interprofessional collaboration in maternal and child care was favorable, although in some cases the interdisciplinary work practice was not effective due to the existence of barriers that hindered its fulfillment.
\end{abstract}

KEY WORDS Prenatal Care; Interprofessional Relations; Primary Health Care; Brazil. 


\section{INTRODUCCIÓN}

El período prenatal es peculiar en la vida de la embarazada, dado que se asimila nueva información y prácticas que culminan en la mejora de los cuidados maternos y del recién nacido. De allí que los programas odontológicos dirigidos a las embarazadas son fundamentales, debido a que es un grupo estratégico, con características psicosociales específicas de la etapa reproductiva, de gran influencia en la educación bucal de sus hijos(1).

El período gestacional está marcado por alteraciones en la salud bucal derivadas de la acción hormonal, así como de náuseas comunes del embarazo, del aumento de la frecuencia de ingesta alimentaria y de la dificultad para la higiene bucal, lo que puede ocasionar modificaciones en la cavidad bucal. Además, las embarazadas suelen asistir menos a las consultas odontológicas debido, principalmente, a creencias y mitos populares que asocian las intervenciones dentales con perjudicar la salud del bebé(2).

La gestación puede ocasionar vulnerabilidades en la salud bucal de la mujer, que dependen de múltiples factores asociados, como los personales (características demográficas y socioeconómicas), comportamentales (prácticas de salud y uso de los servicios) y socioambientales (el propio sistema de salud, por ejemplo) ${ }^{(3,4)}$. Así, aproximadamente el $40 \%$ de las embarazadas presentan enfermedades periodontales ${ }^{(5)}$, en las que un tratamiento adecuado podría tener un impacto positivo ${ }^{(6)}$.

Sin embargo, la práctica de los profesionales en la gestación, el cuidado prenatal y los tratamientos odontológicos presentan divergencias $^{(7)}$. Una investigación brasileña señaló que las y los obstetras, a pesar de conocer las asociaciones existentes entre enfermedades periodontales y parto prematuro, realizaban acciones y orientaciones contrarias e incluso se oponían a conductas como la realización de radiografías, el suplemento con flúor prenatal o el uso de anestésicos locales ${ }^{(5)}$.

Además, la salud bucal de la embarazada repercute en su salud gestacional, por lo que es necesario concientizar, poniendo énfasis en la importancia del uso del servicio odontológico para embarazadas. $Y$, en esta práctica, tanto los cuidadores de la salud materna como los clínicos generales, obstetras y parteras pueden ser elementos colaborativos para este fin $^{(8)}$.

Actualmente, la perspectiva sobre la salud señala la necesidad de comprender e intervenir en las problemáticas del proceso de salud y padecimiento, entendiendo la complejidad creciente del cuidado y de la reestructuración de las prácticas ${ }^{(9)}$. En las organizaciones de salud es esencial la integralidad de las acciones para promover la calidad en la asistencia y reafirmar la práctica interprofesional como modelo de organización de la atención primaria de la salud, buscando atender las demandas de la población en diversos niveles de necesidad ${ }^{(10)}$.

Desde esta lógica, la colaboración interprofesional se sustenta en el equipo de salud, en la reflexión sobre los roles profesionales desempeñados, la resolución de problemas y la negociación de procesos elementales, considerando el conocimiento construido, las singularidades individuales y los diferentes saberes y prácticas profesionales ${ }^{(11)}$.

Además, se destaca la importancia de los equipos de salud familiar, compuestos por múltiples profesionales de diversas categorías: médicos, enfermeros, odontólogos, auxiliares de salud bucal, técnicos en enfermería y agentes comunitarios de salud. Esa noción de interdisciplinaridad en la atención primaria abarca acciones desempeñadas de modo integral e interrelacionado, por profesionales de diversas áreas de conocimiento, desarrolladas de modo creativo, reflexivo y original, buscando superar la fragmentación del conocimiento ${ }^{(12)}$.

Así, la mujer, durante el proceso gestacional presenta un conjunto de características peculiares y múltiples, específicas de esta etapa, que culminan con necesidades multifactoriales y complejas que incluyen dimensiones fisiológicas, psicológicas y comportamentales, sociales y culturales, que exigen acciones en diversos espectros profesionales durante el seguimiento prenatal. Esa multidimensionalidad de exigencias requiere también una 
resolutividad que tenga por objetivo la mejor calidad posible de atención y cuidado, desarrollada para una satisfacción y un bienestar plenos $^{(13,14)}$.

La atención primaria, como pilar estructurante de los sistemas de salud y modelo asistencial principal, requiere de la colaboración interprofesional como estrategia para calificar y potencializar la atención en salud a través de la articulación e integración profesional. Además, el cuidado prenatal como programa estratégico dirigido a la salud reproductiva de la mujer tensiona el desempeño del trabajo en equipo, la valorización de los procesos comunicativos, la acción intersectorial y la atención de demandas oriundas del territorio de la comunidad, lo que resulta en la mejora del acceso universal ${ }^{(5,14,15)}$.

Asimismo, en la búsqueda de integralidad, las relaciones entre los equipos interprofesionales resultan necesarias para alcanzar la calidad del cuidado dirigido a las embarazadas en el sistema de salud. Sin embargo, aún se observan lagunas de conocimiento acerca de las acciones desempeñadas por las categorías profesionales en la Estrategia de Salud Familiar (ESF) de modo integral, considerando las peculiaridades de la mujer, su autonomía y subjetividad. Ante esto, surge la pregunta ¿cómo es la colaboración interprofesional durante el seguimiento odontológico prenatal? Por lo que el objetivo del estudio fue comprender las colaboraciones del equipo interprofesional de salud en el seguimiento odontológico prenatal en atención primaria.

\section{MÉTODO}

Se realizó un estudio exploratorio descriptivo con abordaje cualitativo, realizado en seis unidades de atención primaria de la salud (UAPS) del municipio de Fortaleza, Ceará. Con la participación de gestores, médicos, dentistas y enfermeros, un representante de cada categoría profesional por unidad, que suman un total de 24 trabajadores. Las UAPS fueron seleccionadas por sorteo aleatorio, con representación en cada una de las seis áreas administrativas de la ciudad. Se incluyeron los trabajadores con un mínimo de seis meses de actuación en la UAPS sorteada, y se excluyeron los profesionales que estaban de vacaciones o de licencia en el período de desarrollo de la investigación. La participación de los profesionales fue de acuerdo con la aceptación y la disponibilidad de las y los participantes el día de la visita de la investigadora.

La recolección de información se realizó desde junio a agosto de 2018, por medio de entrevistas individuales semiestructuradas $y$ en profundidad, realizadas en las UAPS según la disponibilidad de los profesionales. La guía de entrevista abordaba las percepciones y prácticas profesionales del equipo en el seguimiento odontológico de embarazadas, focalizando en la colaboración interprofesional.

Para organizar los datos recolectados, se utilizó el análisis de contenido temático, y se identificaron los núcleos de sentido relevantes para el objeto de estudio para la construcción de las categorías ${ }^{(16)}$. El análisis de la información se sustentó en la hermenéutica dialéctica ${ }^{(17,18)}$, para un mejor entendimiento de las relaciones profesionales desarrolladas por los equipos con la intención de proveer asistencia de calidad a las embarazadas.

Se utilizó un código alfanumérico para designar a los participantes: M para médico, E para enfermero, D para dentista y G para gestor, seguido del número correspondiente a cada una de las seis UAPS que sirvieron de escenario para el estudio. La investigación fue aprobada por el comité de ética de la Universidade Estadual do Ceará, bajo el parecer 2.690.258, siguiendo la Resolución 466/2012, del Consejo Nacional de Salud de Brasil ${ }^{(19)}$.

\section{RESULTADOS Y DISCUSIÓN}

De acuerdo con las características sociodemográficas de los participantes de la investigación, se puede observar una media de edad de 42,6 años con variación de 27 a 64 años. La mayoría era del sexo femenino (16) y casada/unión estable (15). 
La formación académica de los seis gestores locales se relacionaba con áreas de administración, pedagogía (2), odontología (2) y enfermería (2), y contaban con estudios de posgrado en áreas relevantes: gestión de servicios en el SUS (2), gestión de personas y gestión en salud (2), y magíster en salud familiar (2). Esas áreas son espacios organizacionales, administrativos y de modelos de gestión importantes para la consolidación y construcción del SUS y de la atención primaria de la salud en Brasil, que repercuten en los modos de composición, organización y producción de salud de la población brasileña ${ }^{(20)}$.

Con relación al año de graduación varió de 1980 a 2016 y el tiempo de trabajo en atención primaria, de dos a 30 años. Se identificó un período mínimo de siete meses y máximo de 25 años de ejercicio en el servicio de salud actual, permanencia que consideramos suficiente para conocer el equipo y los procesos de trabajo.

La lectura del abundante material recolectado y la identificación de los núcleos de sentido importantes para responder a la pregunta de investigación posibilitaron la construcción de tres categorías sobre la atención prenatal: percepciones profesionales acerca de la práctica colaborativa, posibilidades de colaboración interprofesional y prácticas de colaboración en el equipo.

\section{Percepciones profesionales acerca de la práctica colaborativa}

La colaboración interprofesional remite a la esencia de la relación entre los profesionales de distintos campos del conocimiento que posibilita un cuidado en salud más abarcativo $^{(21)}$. Comprende el respeto por las diversas opiniones, el diálogo claro y accesible y la resolución compartida de los problemas ${ }^{(5)}$. De un modo general, todos los profesionales participantes de la investigación consideran la interdisciplinaridad como un requisito fundamental en el cuidado integral y de calidad en la salud perinatal. Ese prestigio emerge en los siguientes discursos:
Creo que es importante [la interdisciplinaridad]. No todo profesional es detentor de todos los saberes, de todo conocimiento pleno, nunca va a ser posible. Hay conocimientos que tienen determinados profesionales, dentro de lo que aprendió, estudió, de sus vivencias; siempre hay algo para sumar en cualquier área del saber, sea relacionado con la salud, la ingeniería, pero siempre alguien tiene algo para aportar. En ese sentido la interdisciplinaridad es importante. El médico contribuye hasta determinado punto, enfermería tiene otra contribución en determinado punto, entonces todo el mundo aporta y al final la contribución es siempre mayor. El benefício para el paciente termina siendo mayor. (M5)

Creo que la interdisciplinaridad es focalizar distintos conocimientos y distintas categorías en el cuidado de un paciente. No quiere decir que voy a dejar de ser dentista, voy a dejar de ser enfermero, voy a dejar de ser médico, sino que voy a tener una mirada con otras categorías para el cuidado de esa embarazada, ¿no? (G6)

Creo que cuando la ven todos los miembros del equipo, termina siendo considerada como un todo. Que la vea un miembro del equipo resulta poco deseable, porque si el seguimiento lo hace solo el enfermero, la cuestión odontológica queda relegada, la cuestión clínica y médica también, entonces necesita ser vista por todos los miembros del equipo. (E6)

El sentido común que observamos en los discursos anteriores podría asimilarse al que Gadamer ${ }^{(17)}$ considera como una orientación de lo que es correcto y verdadero, que busca lo posible, apoyado en vivencias. Sin embargo, se perciben ciertas diferencias en el grupo al preguntar si existe una actuación interdisciplinaria en la unidad de salud donde trabajan:

Tengo un equipo que trabaja perfectamente. Porque, para que veas que me quedo sorprendido, porque no había 
convivido con eso antes, porque muchas veces el médico y el enfermero se ocupan de la atención clínica y la odontología muchas veces hace la derivación por separado, en el consultorio. Pero aquí no, es un trabajo diferente. Hay una integración muy buena. (G2)

De un modo general los equipos en todos los lugares son precarios ¿no? Hablamos de equipo, pero casi no tenemos ese sentimiento de equipo. La odontología está reservada allá en el espacio de ellos, y nosotros aquí. Pero eso es cultural. Y así los equipos por más que sean equipos están muy fragmentados todavía, viste. Como es en todo, todo nuestro ambiente de salud aún está fragmentado. No funciona muy en red. (M5)

Es importante destacar las diferencias de actuación interdisciplinaria dentro de la misma unidad de salud, enfatizando la relevancia de entender los contextos y prácticas ${ }^{(18)}$ de cada servicio de salud. Los siguientes fragmentos de entrevistas son claros respecto de que la interacción sucede cuando el equipo y sus profesionales lo entienden como imprescindible:

Es según la percepción de cada profesional [...] unos más que otros sí, pero a grosso modo, hay una buena interacción. Y como te dije, es una cuestión del propio equipo. No tuve que estar... El equipo estaba empoderado. Con relación a eso, no tuve que decir: Ey gente, vamos a reunirnos. El equipo ya tiene esa consciencia, los profesionales de hecho son muy buenos, creo que es muy, muy bueno. (G5)

Debería haber, debería ser más, más [...] Debería haber una interdisciplinaridad más profundizada sabés, los profesionales realmente actuando en conjunto, y por lo menos aquí eso no sucede. Pero no me preguntes por qué. Creo que eso depende mucho del equipo, de que vea eso como importante. Aquí están muy distanciados, cada uno más en su área y la demanda clínica aquí es muy grande, entonces se sobrecarga bastante y ahí lo dejamos de lado, se termina dejando esa parte de lado. ¿Entendés? (D2)

Estos relatos refuerzan la importancia de la percepción del equipo y de cada miembro sobre la contribución necesaria para una colaboración interprofesional exitosa y en la superación de obstáculos relacionados con la seguridad, el liderazgo y la jerarquía ${ }^{(22)}$. En ese sentido, para Supper et al. ${ }^{(23)}$ la subjetividad de la percepción del ser humano acerca de la realidad y de la conducta profesional, en ciertos momentos, es la forma de mejorar el cuidado en salud y construir nuevas prácticas de relaciones interprofesionales.

Cabe destacar que Minayo ${ }^{(16)}$ considera la inexistencia de una oposición entre la subjetividad y la objetividad. Entiende que esos dos términos se relacionan: la acción es la objetividad y el resultado de la acción es la subjetivación. En el contexto de esta investigación, la realización del trabajo interdisciplinario es la objetividad de los significados que los profesionales atribuyen, y el resultado de esta acción es la propia subjetivación. Se observó que la heterogeneidad también está inserta en las diferentes formas de interacción relatadas por los sujetos entrevistados con relación a las categorías profesionales:

\footnotetext{
Aquí tenemos una interacción mayor con el enfermero y el dentista que con el médico. Ellos están súper ocupados y no tenemos contacto, por eso yo hablé solo con la enfermera. (D6)
}
...lo que funciona aquí es solo la aten- ción del médico y del enfermero. Es muy difícil que hagamos este trabajo junto a los profesionales de odontología. (E4)
...lo que consigo hablar de la interdisci- plinaridad es más de mi trabajo en con- junto con mi enfermera. (M4)

La interacción de la categoría médica y de enfermería fue la más comúnmente planteada; y los cirujanos-dentistas, los menos 
participativos en el proceso interdisciplinario, como se evidencia en los siguientes testimonios:

Ellos también lo dicen, los agentes de salud comentan mucho que los dentistas están muy separados, que están allá en su espacio. [...] Y ni sé si los atienden porque no tengo retorno, ¿me entendés? No tengo un retorno que diga: fulano, de nuestra área, fue atendido así y así. No tengo retorno, un flujo, ni una referencia y ninguna contrarreferencia. ¡No sabemos! Es como si estuviésemos aislados ¿entendés? (E4)

Existe más interacción entre los médicos y los enfermeros. Se ha discutido mucho esa cuestión de que el dentista, por sí mismo, no interactúe mucho con los otros profesionales y, al final, la embarazada está más en manos del médico y la enfermera, médico y enfermera. [...] Entonces, desgraciadamente aquí no tenemos un equipo tan multidisciplinario. Tenemos tres profesionales y termina limitándose a dos. (G3)

Tales relatos reflejan un contexto histórico de la formación y profesionalización de los cirujanos-dentistas que puede influir significativamente en su conducta. Según Ferreira et al.(24), la tecnificación de la odontología entre los años 1930 y 1970 reflejó el modelo tecnicista y organicista basado en el modelo biomédico de atención en salud, pautado en aspectos relativos a la asistencia curativa, superespecialización y proceso de saludenfermedad estático y, desde allí, influyó en el área de enseñanza-aprendizaje, formación y práctica profesional del cirujano-dentista, que se refleja en la individualidad y la poca o ninguna integración con el equipo de salud.

En ese escenario, en los últimos años se ha producido una mayor integración del cirujano-dentista en el equipo, pero aún hay mucho que avanzar. Algunos estudios han mostrado que un deficiente trabajo en equipo puede repercutir negativamente en la atención ofertada en atención primaria ${ }^{(25,26)}$.

\section{Desafíos para la colaboración interprofesional}

El modelo profesional fundamentado en demarcaciones rígidas entre las categorías puede contribuir a la diferenciación profesional inflexible, que acentúa actitudes territoriales y disciplinares en el equipo. Eso puede configurar un importante impedimento para efectivizar un trabajo colaborativo, que resulte en una perspectiva contraria al trabajo interdisciplinario(5).

Se suman cuestiones como el modelo de gestión, las intensas demandas de clínica y de gestión, los pocos profesionales y la dificultad para encontrar momentos de reunión con el equipo de salud, como revelan los relatos de los entrevistados:

Creo que desgraciadamente no tenemos mucho tiempo para reunirnos. A veces, queremos hacer una reunión con todo el mundo, sentarnos y acordar algunas cosas ¿no? Ver cómo es que queda la cuestión de la conducta y todo, pero desgraciadamente no lo conseguimos. Lo hacemos de un modo, el otro profesional lo hace de otro que no sabemos cómo es, y queda esa situación... (E3)

Hago evaluación, como está instituido en Fortaleza. En un horario diferenciado para dar una mayor accesibilidad a la población, hasta incluso para las cuestiones agudas, se quebró la cuestión de los equipos, de reunión, de consulta, de diálogo con sus respectivas poblaciones. [...] pero no es solo el dentista. Es un proceso de salud como un todo y el equipo está fragmentado. (G6)

No tenemos médicos en todos los equipos. [...] Porque tenemos cinco equipos y dos médicos. Ellos realmente no tienen tiempo. [...] Actualmente, tenemos un problema en el auditorio que está condenado con filtraciones y nos condenaron. Estamos sin espacio para reuniones. (D6) 
Los relatos de la mayoría de los trabajadores de la salud entrevistados dejan entrever ciertas deficiencias en la realización de reuniones del equipo de salud. Tal recurso configura una estrategia fundamental en el alcance de la interdisciplinaridad. Los resultados encontrados se condicen con el estudio de Faquim y Frazão ${ }^{(5)}$, en el cual la mayor parte de los profesionales no hace uso de herramientas como las reuniones de planificación y discusión de casos para organizar el trabajo colaborativo en la construcción del cuidado.

La ausencia de encuentros del equipo contribuye al desarrollo de aspectos como el desconocimiento de las competencias y experiencias de otros profesionales. La cultura profesional, la resistencia en establecer comunicación con otros profesionales y la restricción de tempo son factores que pueden dificultar la colaboración efectiva en el equipo de salud ${ }^{(25)}$. Al respecto, presentamos algunos de los relatos relacionados con la existencia y relevancia de la comunicación en el equipo de salud en el cuidado perinatal:

Creo que es un tanto deficiente. Vamos a plantearlo de este modo: algunos años atrás existía la ronda de conversación y ya no la tenemos. Pienso hasta en rescatarla. Para eso estamos pensando en agarrar un día de la agenda y reducir el número de turnos para que podamos hacer esa ronda. Pero sí creo que existe una comunicación puntual. Hay una embarazada " $x$ " que la enfermera o el médico cree que necesita una atención especial. Ellos tienen libre comunicación entre ellos, pero de un modo general creo que desgraciadamente tenemos una deficiencia ¿no? (G1)

Sí, hay, porque incluso ese médico que no tiene el perfil de salud familiar, que suele suceder, incluso aquel que no está relacionado al cuidado prenatal no hay cómo no tener esa integración. Porque, por ejemplo, si le pido el examen de la embarazada y detecto que está con VDRL positivo, reactivo. Entonces tengo que ir a buscar al médico. Es una prioridad esa embarazada. Entonces, me acerco y converso: "Mirá esto, acá dio eso así, así, así, quedó $A B S$, así". Ahí él ya realiza las acciones necesarias, después ella regresa conmigo. Entonces estamos así, caminando dentro del equipo ¿entendés? (E5)

Una comunicación deficiente al interior del equipo fue uno de los hallazgos frecuentes en los relatos de los entrevistados de este estudio. Para la hermenéutica que lidia con la comunicación usual de las relaciones sociales y del sentido común, el ser humano es finito y limitado en su historia y en su lenguaje, que se complementa a través de la comunicación, y debe ser comprendido en su contexto histórico y cultural| ${ }^{(17)}$. La práctica dialéctica complementa esta perspectiva, aportando el dinamismo y la contrariedad de las relaciones sociales como fundamento del proceso comunicativo ${ }^{(16,18)}$.

En el ámbito de la actuación profesional durante el embarazo, ciertos estudios evidencian las relaciones de los cuidados bucales y su repercusión en la salud general, e incluso los efectos de condiciones precarias de salud bucal materna para el futuro bebé. Entre ellos, se destacan las enfermedades periodontales como factor de riesgo para el parto prematuro, principalmente debido a su presencia entre el $5 \%$ y el $20 \%$ de las mujeres embarazadas, pudiendo alcanzar cerca del $40 \%(5,27,28,29,30)$.

Además, los relatos de los participantes concordaron respecto de la importancia de la comunicación y la presencia de las diversas categorías en el equipo de salud. Sin embargo, reconocieron la deficiencia en la práctica de este instrumento en el proceso de trabajo de la unidad de salud. Los resultados de nuestro trabajo discrepan del estudio de Matziou et al. ${ }^{(31)}$ quienes encontraron que los médicos no reconocían el trabajo del enfermero. En este sentido, los sujetos que viven en una realidad específica y que pertenecen a grupos, clases o segmentos sociales distintos, de acuerdo con el momento histórico, pueden tener al mismo tiempo intereses comunes que los nuclean e intereses singulares que los contraponen ${ }^{(16)}$. 
La inexistencia de la relación interprofesional puede producir una mayor cantidad de fallas en la asistencia a los usuarios del servicio, fortaleciendo la necesidad del reconocimiento de esa relación por todos los miembros del equipo ${ }^{(16)}$. Esta pertenencia se evidencia en estos relatos:

Considero súper importante la cuestión de que todos los profesionales sepan su rol y lo sepan desempeñar también. Y aquí contamos con la participación de nuestro equipo, el médico, la enfermera, los agentes comunitarios de salud, los técnicos de enfermería que cada equipo tiene el suyo; y el dentista del equipo también. $Y$ con relación a la importancia de cada uno, es extrema, porque tengo que saber hasta dónde puedo ir, hasta dónde va el rol del médico, hasta dónde es importante el técnico de enfermería, que generalmente es quien hace la primera admisión y el agente de salud que hace el seguimiento en el domicilio y también en la unidad de salud. Así, la importancia de cada uno es $100 \%$. (E1)

Es importante, es bueno que el equipo tenga tanto el seguimiento, como la visión de profesionales que, aunque trabajan en el área de salud, son de sectores diferentes. Por ejemplo, como médicos tenemos una cuestión muy así, una visión, a veces a causa de la facultad, una visión muy medicalizante todavía. (M4)

El relato anterior muestra la fragmentación histórica de las matrices curriculares en la formación de grado, que aún está presente incluso con innumerables discusiones y evidencias de la calidad superior del trabajo interdisciplinario comparado al trabajo segregado ${ }^{(25,26,31,32)}$. Del mismo modo, Faquim y Frazão(5) observaron en su investigación que las diferencias en las actitudes de colaboración interprofesional fueron más evidentes entre unidades de atención primaria al comparar la categoría profesional, y mostraron que la forma de relacionarse en el equipo puede superar la formación fragmentada existente en las instancias de grado.
El desarrollo de un trabajo colaborativo y efectivo propicia la percepción de la interdependencia entre los profesionales, la concordancia entre áreas interdisciplinarias, las prácticas multiprofesionales en las que se pueda compartir y colaborar, beneficiando a todos los involucrados: cuidadores, cuidados y el sistema de salud como un todo ${ }^{(32)}$.

\section{Prácticas de colaboración en el equipo}

Los discursos denotan la relevancia del Sistema Único de Salud (SUS) y de la estrategia de salud familiar en el cuidado de la población y su relación con una mirada ampliada de la salud. Al entender el cuidado de la salud materno-infantil de forma contextualizada, atravesado por un proceso social, se estaría contribuyendo a la construcción de una salud colectiva mediada por la vinculación de prácticas de promoción, prevención, recuperación y rehabilitación, con la integración de las disciplinas de salud y de ciencias sociales, planificación, gestión y epidemiología analítica en busca de un objetivo: la salud ${ }^{(33)}$. Esta importancia surge en los relatos de los participantes de la investigación:

\begin{abstract}
Siento lo siguiente: la salud de la familia es un..., cómo decirlo..., no es un proyecto, sino un proceso muy importante en nuestra población que necesita realmente del SUS, nuestra población carente. Entonces, esa visión más amplia de la atención creo que es muy válida. (D2)
\end{abstract}

...pero es de fundamental importancia, diversas miradas. El Programa de Salud Familiar es eso. Mejora esa cuestión de la pluralidad de visiones, de profesionales, sobre todo en lo que se refiere a la embarazada. (G5)

La atención prenatal realizada con la unión de diversos saberes, desde la perspectiva de un cuidado integral, posibilita una asistencia humanizada y de calidad, brinda la oportunidad del desarrollo de prácticas educativas, la construcción del vínculo y la integración 
entre los profesionales del equipo ${ }^{(34,35)}$. Siendo así, las prácticas educativas interdisciplinarias realizadas en el ámbito de las acciones del Programa de Salud Familiar son consideradas estrategias significativas para el alcance de la interdisciplinaridad, de un cuidado integral y humanizado:

Teníamos grupos de embarazadas, y en ese grupo de embarazadas actuaban varios profesionales de distintas áreas, no solo de salud. A veces convocábamos a quienes considerábamos que hacía falta, a veces al médico, a veces a la enfermera, a la asistente social, para conversar sobre diversos asuntos y teníamos el cronograma bien hechito de las reuniones que teníamos con la embarazada. Y era muy interesante ver esa interacción y cómo conseguíamos pasar información muy buena para ellas. Y nos sentíamos realizados porque ese es nuestro trabajo, no solo curamos, no hacemos solo tratamiento. (D2)

Creo que solo vamos a conseguir eso [interdisciplinaridad] si hacemos un grupo ¿entendés? Porque en la consulta clínica cada uno hace lo suyo. El médico en su consultorio, la enfermera en el de ella y el dentista en el de él, y la nutricionista también. Pero es esa cosa de compartir el saber, de estar juntos, entonces en ese momento creo que vamos a conseguir hacer esa atención interdisciplinaria, que es tan importante en realidad. (G4)

Los relatos muestran cuán impactantes son para los profesionales los momentos colectivos para el alcance de la interdisciplinaridad. Tales encuentros proporcionan sentimientos de satisfacción profesional en cuanto a conseguir realizar su práctica laboral de acuerdo con lo que considera correcto, lo que lo hace sentirse bien por estar contribuyendo, de hecho, con el empoderamiento de la embarazada en el autocuidado, además de estar interactuando con las demás categorías, intercambiando experiencias y saberes. En contrapartida, se observó un sentimiento nostálgico de percibir que estas prácticas se perdieron con el tiempo.

Desde esta perspectiva, el embarazo consiste en un momento único para la mujer y su salud bucal, se destaca como un punto crucial para el bienestar y la calidad de vida; sin embargo, es relegada a un segundo plano en comparación con otros aspectos abordados en la atención asistencial materna, como el examen obstétrico, la medición antropométrica, las orientaciones en cuanto al uso de medicamentos, entre otros cuidados ${ }^{(34,36)}$.

En el período de embarazo, las alteraciones del comportamiento más frecuentes, asociadas a la deficiente higiene bucal, predisponen al desarrollo de lesiones cariosas que pueden evolucionar a importantes procesos inflamatorios e infecciosos. Sumado a esto, las náuseas con vómitos frecuentes durante el primer trimestre de gestación pueden acarrear una mayor actividad bacteriana debido a la reducción del $\mathrm{pH}$ bucal consecuente de la exposición a los ácidos gástricos ${ }^{(2,37)}$.

Además, se destaca que ciertas contribuciones al cuidado de la salud bucal en embarazadas son deficientes, como mencionan Gonçalves y Sonza ${ }^{(38)}$ en su investigación, en la que evidenciaron que un número considerable de cirujanos-dentistas $(41,7 \%)$ no participaba y no realizaba actividades educativas en grupos de embarazadas debido a la gran demanda clínica, por falta de invitación o incluso por incompatibilidad horaria para su participación. Tal hallazgo denota la ausencia de una planificación interdisciplinaria en el servicio de salud.

En este sentido, si bien se observa que ciertos profesionales aceptan los procesos educativos y promotores de la salud, existen barreras que impiden efectivizar las prácticas educativas interdisciplinarias:

La educación en salud es un trabajo de hormiguita, no ves un resultado inmediato. Los gestores reclaman resultados inmediatos, ellos reclaman números porque es lo que ellos pueden presentar. No tenés cómo cuantificar eso. Es un trabajo cualitativo. A veces vas a trabajar en promoción de la salud y los gestores: 
“¿dónde están los números?". "No tenemos números". "¿Qué hacen?, ¡no están haciendo nada!" Entonces ¿no es necesario un profesional para la prevención y promoción de la salud?, ¿no? A corto plazo genera esa sensación, pero a largo plazo ellas aprenden sobre el cuidado de sí, a trabajar en la prevención. Las personas perdieron mucho la noción del autocuidado; entonces, deben apropiarse de determinados conocimientos. Creo que determinados conocimientos no deben quedar confinados a categorías, a determinadas personas. Hay conocimientos que deben ser distribuidos a todos ¿no? para que ellas puedan apropiarse de eso y el beneficio sea mayor. (M5)

...Tres equipos en una población de 43 mil, eso no es lo ideal y termina haciendo que el servicio no sea ofertado como debería ser. (G2)

Los relatos expresan el interés de los profesionales por realizar una atención interdisciplinaria, asociada a una desmotivación, debido a la gran demanda y al poco tiempo de la consulta; además del reclamo "cuantitativista" de la gestión, que dificulta efectuar un cuidado integral e interdisciplinario. Siendo así, la reflexión debe apoyarse sobre una determinada realidad, que considere el contexto histórico de los sujetos involucrados y sus prácticas laborales, ya que los discursos son expresiones de su tiempo y de su cultura(16,18).

En este ámbito, el contexto histórico, político y social contribuye a explicar el significativo desinterés en la entrevista: el aumento de los ataques políticos al SUS ocurridos en los últimos años, que intentan desvalorizar el sistema de salud para beneficiar a la clase política-empresarial brasileña.

Es importante destacar la construcción del vínculo como una herramienta significativa en los procesos de corresponsabilización y de confianza de la embarazada en el equipo de salud; enlace que se ve fortalecido en los procesos interdisciplinarios individuales/colectivos de educación permanente en salud. Ese contexto favorece la formación de una espiral de salud a través de la creación del vínculo equipo de salud-embarazada, equipo de salud-madre y compañero; equipo de salud-madre y bebé; equipo de salud-niñez; equipo de salud-adolescente y, de forma continua, humanizada y natural, la integración de la nueva familia generada y el proceso de vinculación de generación en generación ${ }^{(28)}$. Todo ese proceso debe fortalecerse dentro del equipo de salud, como muestran los siguientes testimonios:

Entonces, cuando existe ese vínculo del equipo, esa idea del Programa de Salud Familiar, eso se traslada a la paciente. Conoce a su dentista, conoce a su médico. Entonces, cuando llega a la consulta prenatal con el médico, el enfermero y el dentista, se consigue trabajar mejor. Ella recibió orientación [...] Entonces, cuando los equipos se fortalecen, por el propio vínculo terminás referenciando y las personas comienzan a entender cómo funciona la atención. (G2)

$Y$ es siempre bueno porque terminamos enganchándonos, porque sabemos que está el compañero, también. Incluso, hasta en el cuaderno de la embarazada hay una parte para que completemos. Si él se hace un análisis de sangre, se ve cómo está la salud de él. Y tienen también hasta la parte odontológica. Está lo de él también en el costadito. En el caso que sea evaluado. ¡Eso es maravilloso! Yo aprovecho y engancho a los chicos también y pregunto: “¿Cómo está la salud de los chicos, a alguno le duele un diente? ¿Hace tiempo que no van al dentista?" En fin, sabemos que es así la salud de la familia, entonces tenemos que aprovechar al máximo. Entonces, el día que viene la embarazada, viene la madre de ella, vienen los hijos y aprovechamos y ya intentamos todo ahí. (E3)

En las estrategias interdisciplinarias, el eje de educación en salud es muy fuerte en los momentos de sensibilización individuales o colectivos. La percepción del profesional acerca 
de que la interdisciplinaridad es esencial, marca una diferencia en su conducta durante la atención prenatal. Santos Neto et al. ${ }^{(39)}$ señalan el período gestacional como una oportunidad importante para la realización de estos encuentros, ya que las mujeres embarazadas están más receptivas a la nueva información y son valiosas promotoras de salud en los microprocesos educativos familiares. El potencial multiplicador de la mujer embarazada está bien ejemplificado en el siguiente relato:

Creo que más allá de la salud en sí, está la cuestión de la cultura, de la concientización, que la madre ya sale mucho más concientizada y será una multiplicadora en su familia. Va a transmitir eso al hijo más viejo, tal vez para el compañero, para el esposo y ahí se va multiplicando la idea de que es necesario de hecho. (G1)

El discurso del participante G1 platea la cuestión cultural como condicionante de la percepción. En un estudio realizado por Costa et al. ${ }^{(26)}$ se encontró que los profesionales relacionaron la resolutividad en la atención primaria a las acciones de un equipo interprofesional, que suma diferentes saberes, construye vínculos basados en el entendimiento del trabajo del otro, integra a los profesionales y valoriza la contribución de cada miembro en la oferta del cuidado, lo que da como resultado una asistencia a la salud más resolutiva. Sin embargo, Morgan, Pullon y McKinlay ${ }^{(40)}$ consideran la colaboración interprofesional como una práctica aún distante en las rutinas de trabajo en la atención primaria.

Esas narrativas encontradas en la investigación posibilitan un mejor entendimiento acerca de las cuestiones relacionadas con la colaboración interprofesional en las prácticas del equipo de atención primaria, con foco especial en la atención odontológica para las embarazadas.

\section{CONSIDERACIONES FINALES}

La colaboración interprofesional está atravesada por cuestiones que permean los conceptos individuales de interdisciplinaridad, roles profesionales de las categorías presentes en la atención primaria y gestiones participativas de los servicios de salud. Se destaca la importancia de las discusiones acerca de las relaciones entre los distintos trabajadores involucrados en el proceso de asistencia a mujeres en el cuidado prenatal, especialmente, en la perspectiva de la atención odontológica.

Las prácticas profesionales que involucran el seguimiento odontológico prenatal son similares en los escenarios estudiados, en los cuales aún hay una necesidad de mayor interacción entre cirujanos-dentistas y otros integrantes del equipo, en la tentativa de calificar la asistencia ofrecida a las embarazadas.

Además, la visión acerca de la salud bucal y de su inserción en la atención primaria necesita ser ampliada, con la intención de mejorar las prácticas desarrolladas por todos los integrantes del equipo, desde una actuación que posibilite la integralidad en la asistencia de las embarazadas. Las especificidades del escenario se plantean como limitantes del estudio; sin embargo, se destaca que las prácticas encontradas tal vez no difieran de otros servicios del país, por ser constituidas a partir de normativas, dificultades y potencialidades similares del mismo sistema de salud.

\section{REFERENCIAS BIBLIOGRÁFICAS}

1. Carvalho JAM, Codato LAB, Carmona $\mathrm{OH}$, Papi RC, Sahyun RE, Garrido DM, et al. Avaliação do acesso de gestantes à atenção odontológica realizada pelo grupo PET-Saúde da Universidade Estadual de Londrina-PR. Revista da ABENO. 2014;14(1):81-86.
2. Ferreira SMSP, Pinheiro ES, Silva RV, Silva JF, Batista LD, Fernandes CG. Pré-natal odontológico: acessibilidade e ações ofertadas pela atenção básica de Vitória da Conquista-BA. Revista da Faculdade de Odontologia de Lins. 2016;26(2):3-16. doi: 10.15600/2238-1236/fol.v26n2p3-16.

3. Johnson M, George A, Dahlen H, Ajwani S, Bhole S, Blinkhorn $A$, et al. The midwifery ini- 
tiated oral health-dental service protocol: an intervention to improve oral health outcomes for pregnant women. BMC Oral Health. 2015;15(2). doi: 10.1186/1472-6831-15-2.

4. Kateeb E, Momany E. Dental caries experience and associated risk indicators among Palestinian pregnant women in the Jerusalem area: a cross-sectional study. BMC Oral Health. 2018;18(1):170. doi: 10.1186/s12903-018-0628-x.

5. Faquim JPS, Frazão P. Percepções e atitudes sobre relações interprofissionais na assistência odontológica durante o pré-natal. Saúde Debate. 2016;40(109):59-69.

6. Musskopf ML, Milanesi FC, Rocha JM, Fiorini $\mathrm{T}$, Moreira $\mathrm{CHC}$, Susin C. Oral health related quality of life among pregnant women: a randomized controlled trial. Brazilian Oral Research. 2018;32:e002. doi: 10.1590/1807-3107bor-2018. vol32.0002.

7. Jackson JT, Quinonez RB, Kerns AK, Chuang A, Eidson RS, Boggess KA, et al. Implementing a Prenatal Oral Health Program Through Interprofessional Collaboration. Journal of Dental Education. 2015;79(3):241-248.

8. George A, Dahlen HG, Blinkhorn A, Ajwani S, Bhole $S$, Ellis $S$, et al. Evaluation of a midwifery initiated oral health-dental service program to improve oral health and birth outcomes for pregnant women: A multi-centre randomised controlled trial. International Journal of Nursing Studies. 2018;82:49-57. doi: 10.1016/j.ijnurstu.2018.03.006.

9. Arruda LS, Moreira COF. Colaboração interprofissional: um estudo de caso sobre os profissionais do Núcleo de Atenção ao Idoso da Universidade Estadual do Rio de Janeiro (NAI/UERJ), Brasil. Interface: Comunicação, Saúde, Educação. 2018;22(64):199-210.

10. Agreli HLF. Prática interprofissional colaborativa e clima do trabalho em equipe na Atenção Primária à Saúde. [Tese]. São Paulo: Faculdade de Enfermagem, Universidade de São Paulo; 2017.

11. Araújo TAM, Vasconcelos ACCP, Pessoa TRRF, Forte FDS. Multiprofissionalidade e interprofissionalidade em uma residência hospitalar: o olhar de residentes e preceptores. Interface: Comunicação, Saúde, Educação. 2017;21(62):601-613.

12. Loch-Neckle G, Seemann G, Eidt HB, Rabuske MM, Crepaldi MA. Desafios para a ação interdisciplinar na atenção básica: implicações relativas à composição das equipes de saúde da família. Ciência e Saúde Coletiva. 2009;1(Supl. 1):14631472.
13. Souza ZNR, Rosa MC, Bastiani JAN. Maternidade: percepções de gestantes primíparas usuárias do Serviço Básico de Saúde. Journal of the Health Sciences Institute. 2011;29(4):272275 .

14. Peduzzi M, Agreli HF. Teamwork and collaborative practice in Primary Health Care. Interface: Comunicação, Saúde, Educação. 2018; 22 (Supl. 2):1525-1534.

15. Arruda GMMS, et al. O desenvolvimento da colaboração interprofissional em diferentes contextos de residência multiprofissional em Saúde da Família. Interface: Comunicação, Saúde, Educação. 2018;22(Supl. 1):1309-1323.

16. Minayo MCS. O desafio do conhecimento: pesquisa qualitativa em saúde. 14 ed. São Paulo: Hucitec Editora, 2014.

17. Gadamer HG. Verdade e método. Petrópolis: Vozes, 1999.

18. Habermas J. Dialética e hermenêutica. Porto Alegre: LPM, 1987.

19. Conselho Nacional de Saúde (Brasil). Resolução 466, de 12 de dezembro de 2012. Aprova as diretrizes e normas regulamentadoras de pesquisas envolvendo seres humanos. Diário Oficial da União. 12 dez 2012.

20. Ravioli AF, Soárez PC, Scheffer M C. Modalidades de gestão de serviços no Sistema Único de Saúde: revisão narrativa da produção científica da Saúde Coletiva no Brasil (2005-2016). Cadernos de Saúde Pública. 2018;34(4):e00114217.

21. Matuda CG, Pinto NRS, Martins CL, Frazão P. Colaboração interprofissional na Estratégia Saúde da Família: implicações para a produção do cuidado e a gestão do trabalho. Ciência e Saúde Coletiva. 2015;20(8):2511-2521.

22. Van Schaik SM, O ' brien BC, Almeida SA, Adler SR. Perceptions of interprofessional teamwork in low-acuity settings: a qualitative analysis. Medical Education. 2014;48(6):583-592.

23. Supper I, Catala O, Lustman M, Chemla C, Bourgueil Y, Letrilliart L. Interprofessional collaboration in primary health care: a review of facilitators and barriers perceived by involved actors. Journal of Public Health. 2015;37(4):716-727.

24. Ferreira NP, Ferreira AP, Freira MCM. Mercado de trabalho na Odontologia: contextualização e perspectivas. Revista de Odontologia da UNESP. 2013;42(4):304-309. 
25. Fewster-Thuente L, Velsor- Friedrich B. Interdisciplinary collaboration for healthcare professionals. Nursing Administration Quarterly. 2008;32(1):4048.

26. Costa JP, Jorge MSB, Vasconcelos MGF, Paula ML, Bezerra IC. Resolubilidade do cuidado na atenção primária: articulação multiprofissional e rede de serviços. Saúde Debate. 2014;38(103):733743. doi: 10.5935/0103-1104.20140067.

27. Gonçalves JB, Guimarães ALA, Araújo TLC, Amaral RC. Conhecimento sobre saúde bucal das gestantes atendidas em CRAS. Interfaces. 2015;3(8):1-8.

28. Bastos RDS, Silva BS, Cardoso JA, Farias JG, Falcão GGVCS. Demystifying dental care pregnant women: literature review. Journal of Dentistry and Public Health. 2014;5(2):104-116. doi: 10.17267/2596-3368dentistry.v5i2.272.

29. Muwazi L, Rwenyonyi1 CM, Nkamba M, Kutesa A, Kagawa M, Mugyenyi G, Kwizera G, Okullo I. Periodontal conditions, low birth weight and preterm birth among postpartum mothers in two tertiary health facilities in Uganda. BMC Oral Health. 2014;14:42. doi: 10.1186/1472-6831-14-42.

30. Shah M, Muley A, Muley P. Effect of nonsurgical periodontal therapy during gestation period on adverse pregnancy outcome: a systematic review. The Journal of Maternal-Fetal and Neonatal Medicine. 2013;26(17):1691-1695. doi: 10.3109/14767058.2013.799662.

31. Matziou V, Vlahioti E, Perdikaris P, Matziou T, Megapanou E, Petsios K. Physician and nursing perceptions concerning interprofessional communication and collaboration. Journal of Interprofessional Care. 2014;28(6):526-533. doi: 10.3109/13561820.2014.934338.

32. Nancarrow SA, Borthwick AM. Dynamic professional boundaries in the healthcare workforce.
Sociology of Health and Illness. 2005;27(7):897919. doi: 10.1111/j.1467-9566.2005.00463.x.

33. Souza LEPF. Saúde Pública ou Saúde Coletiva? Revista Espaço para a Saúde. 2014;15(4):7-21.

34. Anversa ETR, Bastos GAN, Nunes LN, Pizzol TSD. Qualidade do processo de assistência prénatal: unidades básicas de saúde e unidades de Estratégia Saúde da Família em município no Sul do Brasil. Cadernos de Saúde Pública. 2012;28(4):789800. doi: 10.1590/S0102-311X2012000400018.

35. Melo MCP, Coelho EAC. Integralidade e cuidado a grávidas adolescentes na Atenção Básica. Ciência e Saúde Coletiva. 2011;16(5):2549-2558.

36. Simão AMS, Santos JLG, Erdmann AL, Mello ALSF, Backes MTS, Magalhães ALP. Gestão do cuidado de enfermagem pré-natal num Centro de Saúde de Angola. Revista Brasileira de Enfermagem. 2019;72(Suppl 1):136-143.

37. Piscoya MDBV, Ximenes RAA, Silva GM, Jamelli SR, Coutinho SB. Periodontitis-associated risk factors in pregnant women. Clinics. 2012;67(1):27-33. doi: 10.6061/clinics/2012(01)05.

38. Gonçalves PM, Sonza QN. Pré-natal odontológico nos postos de saúde de Passo Fundo/RS. Journal of Oral Investigations. 2018;7(2):20-32. doi: 10.18256/2238-510X.2018.v7i2.2727.

39. Santos Neto ET, Oliveira AE, Zandonade E, Leal MC. Acesso à assistência odontológica no acompanhamento pré-natal. Ciência e Saúde Coletiva. 2012;17(11):3057-3068.

40. Morgan S, Pullon S, McKinley E. Observation of interprofessional collaborative practice in primary care teams: an integrative literature review. International Journal of Nursing Studies. 2015;52(7):12171230. doi: 10.1016/j.ijnurstu.2015.03.008. 